\title{
Graphic and Protographic Lists of Integers
}

\author{
Dmitry Fon-Der-Flaass* \\ Institute of Mathematics \\ Novosibirsk State University, Novosibirsk, Russia \\ flaass@math.nsc.ru \\ Douglas B. West ${ }^{\dagger}$ \\ Department of Mathematics \\ University of Illinois, Urbana, IL 61801 \\ west@math.uiuc.edu
}

Submitted: May 19, 2003; Accepted: Dec 21, 2003; Published: Jan 2, 2004

MR Subject Classifications: $05 \mathrm{C} 07$

\begin{abstract}
A positive list (list of positive integers) is protographic if its merger with all but finitely many positive graphic lists is graphic. Define the family $\mathcal{P}_{s}$ of $s$-protogaphic lists by letting $\mathcal{P}_{0}$ be the family of positive graphic lists and letting $\mathcal{P}_{s}$ for $s>0$ be the family of positive lists whose merger with all but finitely many lists in $\mathcal{P}_{s-1}$ is in $\mathcal{P}_{s-1}$.

The main result is that $X \in \mathcal{P}_{s}$ if and only if $t(X) \in \mathcal{P}_{s-1}$, where $t(X)$ is the list obtained from $X$ by subtracting one from each term of $X$ (deleting those that become 0 ) and appending a 1 for each term of $X$. A corollary is that the maximum number of iterations to reach a graphic list from an $n$-term even list with sum $2 k$ is $k-n+1$ (when $k \geq n$ ), achieved by the unique such list having one term larger than 1 .
\end{abstract}

\section{Introduction}

An integer list of length $n$ is an $n$-tuple of integers. A graphic list is a list whose entries are the degrees of the vertices in a simple graph. Whether a list is graphic is determined by the multiset of entries; the order of the entries is irrelevant. Because entries equal to 0

${ }^{*}$ This work partially supported by RFFI grants 03-01-00796 and 02-01-00039 and by grant No.6 of the 6th Expertise of Young Scientists' Project of RAN.

${ }^{\dagger}$ This material is based upon work supported by NSA under Award No. MDA904-03-1-0037, which requires the disclaimer that any opinions, findings, and conclusions or recommendations expressed in this publication are those of the author(s) and do not necessarily reflect the views of the NSA. 
do not affect whether a list is graphic, we consider only lists of positive integers. (We use "list" rather than "sequence" since a sequence is a function whose domain is infinite.)

Many characterizations of graphic lists are known: Sierksma and Hoogeveen [6] state seven. A well-known explicit characterization due to Erdős and Gallai [2] is that, when the entries $d_{1}, \ldots, d_{n}$ are written in nonincreasing order, the inequalities $\sum_{i=1}^{k} d_{i} \leq k(k+$ $1)+\sum_{i=k+1}^{n} \min \left\{k, d_{i}\right\}$ hold for every $k$ (see Aigner and Triesch [1] for an elegant proof).

This note introduces a measure of how far a list is from being graphic. Let a positive list $X$ be protographic if there are only finitely many positive graphic lists $Y$ such that the merger $X \cup Y$ is not graphic, where the merger of two lists is obtained by summing the multiplicities of their elements.

More generally, define a sequence of families of lists recursively as follows. Let $\mathcal{P}_{0}$ be the set of positive graphic lists. For $s>0$, let $\mathcal{P}_{s}$ be the set of positive lists $X$ such that $X \cup Y \in \mathcal{P}_{s-1}$ for all but finitely many $Y \in \mathcal{P}_{s-1}$. The lists in $\mathcal{P}_{s}$ are the s-protographic lists. Thus the positive graphic lists are the 0-protographic lists, and the protographic lists are the 1-protographic lists.

As might be expected, every $s$-protographic list is also $(s+1)$-protographic. This follows from the fact that $X \cup Y \in \mathcal{P}_{s}$ when $X \in \mathcal{P}_{s}$ and $Y \in \mathcal{P}_{s}$. To see this latter fact, write $(X \cup Y) \cup Z$ as $X \cup(Y \cup Z)$ for $Z \in \mathcal{P}_{s-1}$. We have $Y \cup Z \in \mathcal{P}_{s-1}$ for all but finitely many such $Z$. Excluding the finitely many $Z$ such that $Y \cup Z \notin \mathcal{P}_{s-1}$ and the finitely many $Z$ such that $Y \cup Z \in \mathcal{P}_{s-1}$ but $X \cup(Y \cup Z) \notin \mathcal{P}_{s-1}$, we have that $(X \cup Y) \cup Z \in \mathcal{P}_{s-1}$ for all but finitely many $Z \in \mathcal{P}_{s-1}$.

We use $\max (X)$ for the largest entry and $\ell(X)$ for the length (number of terms) of a list $X$. Our main result is the characterization of $s$-protographic lists using a special operation on lists. Let $t(X)$ denote the list obtained from $X$ by subtracting 1 from each element of $X$ (discarding terms that reach 0 ) and then appending $\ell(X)$ entries equal to 1 .

We prove that $X$ is protographic if and only if $t(X)$ is graphic. This serves as the basis step for an induction to prove the characterization in general:

Theorem 1 If $X$ is a positive list of integers, and $s$ is a positive integer, then $X \in \mathcal{P}_{s}$ if and only if $t(X) \in \mathcal{P}_{s-1}$.

The definition implies inductively that only lists with even sum can be $s$-protographic. We define an even list to be a positive list with even sum. An even list with all entries equal to 1 is graphic. Since $\max (t(X))=\max (X)-1$ when $\max (X)>1$, our theorem thus proves inductively that every list with even sum belongs to $\mathcal{P}_{s}$ for some $s$.

For an even list $X$, we define the non-graphicality $\gamma(X)$ to be the minimum $s$ such that $X \in \mathcal{P}_{s}$. A corollary of our theorem shows that the maximum non-graphicality among even lists with sum $2 k$ is $k$, achieved by the list consisting of a single term equal to $2 k$. More generally, the maximum non-graphicality among $n$-term even lists with sum $2 k$ is $k-n+1$ (when $k>n-1$ ), achieved by the unique such list having one term larger than 1 . The non-graphicality of $n$-term lists with unbounded sum is unbounded. 


\section{The Proofs}

Let $n(G)$ denote the number of vertices of a graph $G$. The boundary $\partial S$ of a set $S$ of vertices in a graph $G$ is the set of vertices outside $S$ whose neighborhoods intersect $S$. A dominating set for $G$ is a set $S \subseteq V(G)$ such that $\partial S=V(G)-S$. Ore [5] observed that every graph $G$ without isolated vertices has a dominating set of size at most $n(G) / 2$. A simple proof is that for every minimal dominating set, the remaining vertices also form a dominating set.

Lemma 2 If $G$ is a simple graph without isolated vertices such that $|\partial S|<k$ for all $S \subseteq V(G)$, then $n(G) \leq 2 k-2$.

Proof. Let $S$ be a smallest dominating set of $G$. By Ore's observation [5], $n(G)=$ $|S|+|\partial S| \leq n(G) / 2+k-1$. Thus $n(G) \leq 2 k-2$.

A list $X$ with $\max (X) \geq \ell(X)$ is not graphic. The Havel-Hakimi Theorem $([3,4])$ states that a positive list $X$ is graphic if and only if the list obtained from $X$ by deleting the element $\max (X)$ and subtracting 1 from $\max (X)$ of the next largest elements is graphic. Let $X^{\prime}$ denote the positive list obtained from a list $X$ by doing this and also dropping any elements that thus become 0 . We use $1^{k}$ to denote $k$ entries equal to 1 .

We will need an operation on simple graphs that also is used in inductive proofs of the Havel-Hakimi Theorem. Given vertices $w, x, y, z$ in a simple graph $G$ such $w x, y z \in E(G)$ and $x y, w z \notin E(G)$, the operation of deleting $w x, y z$ and adding $x y, w z$ to $E(G)$ is a 2-switch; it produces another simple graph with the same vertex degrees.

Theorem 3 When $X$ is a positive list, $X \in \mathcal{P}_{1}$ if and only if $t(X)$ is graphic.

Proof. Let $k=\ell(X)$.

Necessity. Let $Y_{n}$ be the degree list of the star with $n$ leaves. By the definition of $\mathcal{P}_{1}$, the list $X \cup Y_{n}$ is graphic for sufficiently large $n$, say $n>n_{0}$. Take $n$ such that $n>\max \left\{n_{0}, \max (X), k\right\}$. By the Havel-Hakimi Theorem, $\left(X \cup Y_{n}\right)^{\prime}$ is graphic. Since $n=\max \left(X \cup Y_{n}\right)$, and the next $k$ largest elements are those of $X$, and $n>k$, we have $\left(X \cup Y_{n}\right)^{\prime}=\left(x_{1}-1, \ldots, x_{k}-1,1^{k}\right)=t(X)$ (discarding terms such that $x_{i}-1=0$ ).

Sufficiency. Assume that $t(X)$ is graphic. We claim that if $Y$ is a positive graphic list of length at least $2 k-1$, then $X \cup Y$ is graphic. Since there are finitely many graphic lists of length at most $2 k-2$, this will yield $X \in \mathcal{P}_{1}$.

Among all graphs with degree list $t(X)$, choose one whose set of vertices of degree 1 induces the fewest edges. Let $H$ be the graph with $2 k$ vertices obtained from it by adding an isolated vertex for each 1 in $X$. Let $w_{1}, \ldots, w_{k}$ be $k$ vertices of degree 1 in $H$ that induce the fewest edges among all sets of $k$ vertices of degree 1 . The remaining vertices are $u_{1}, \ldots, u_{k}$, indexed so that $d_{H}\left(u_{i}\right)=x_{i}-1$ (this includes all the added isolated vertices).

Let $W=\left\{w_{1}, \ldots, w_{k}\right\}$ and $U=\left\{u_{1}, \ldots, u_{k}\right\}$. We reduce the problem to the case where $W$ is an independent set in $H$. If $W$ induces an edge, then its endpoints have degree 1 , so if there is an edge induced by $U$ we can perform a 2 -switch to reduce the 
number of edges within $W$. Hence if $W$ induces an edge, then we may assume that $U$ is an independent set in $H$. Now $\sum d_{H}\left(u_{i}\right)<k$, because the only edges incident to $U$ are also incident to $W$, and fewer than $k$ such edges are incident to $W$.

Thus $X$ consists of $k$ positive numbers summing to $k+j$, where $j<k$. In this case we show that $X$ is graphic, by induction on $k$. If all entries are 1 , then $X$ is realized by a matching. Otherwise, the pigeonhole principle implies that $X$ contains a 1 . Form $X^{\prime}$ by deleting this 1 and subtracting 1 from some larger element of $X$. Now $X^{\prime}$ has length $k-1$ and sum $k-1+j-1$, with $j-1<k-1$. By the induction hypothesis, $X^{\prime}$ is graphic, and we add a pendant edge to a realization of it to obtain a realization of $X$. Since every $s$-protographic list is $(s+1)$-protographic, this yields $X \in P_{1}$.

Hence we may assume that $W$ is an independent set in $H$. Now let $G$ be a graph with degree list $Y$. By Lemma 2, there exists $S \subseteq V(G)$ with $|\partial S| \geq k$; give the names $w_{1}, \ldots, w_{k}$ to distinct vertices in $\partial S$. Let $z_{1}, \ldots, z_{j}$ (not necessarily distinct) be vertices of $S$ such that $z_{i} w_{i} \in E(G)$ for each $i$.

Because $W$ is an independent set in $H$, the union $G \cup H$ is a simple graph with $k+\ell(Y)$ vertices. In $G \cup H$, replace the edge $z_{i} w_{i}$ with the edge $z_{i} u_{i}$ for $1 \leq i \leq j$. This increases the degree of $u_{i}$ to $x_{i}$ and decreases the degree of $w_{i}$ to $d_{G}\left(w_{i}\right)$. Hence the modified graph $F$ is a simple graph with degree list $X \cup Y$.

Let $B_{s, n}$ denote the list of length $n$ consisting of one entry equal to $n-1+2 s$ and $n-1$ entries equal to 1 . Note that $B_{0, n}$ is the degree list of a star with $n$ vertices. By construction, it is immediate that $t\left(B_{s, n}\right)=B_{s-1, n+1}$. The proof of the main result (Theorem 1) involves a statement about $B_{s, n}$ equivalent to the other two.

The application of 2-switches in the proof of the Havel-Hakimi Theorem is a statement that we will need here: for every graphic list $X$, there is a simple graph $G$ whose degree list is $X$ in which a vertex of highest degree is adjacent only to vertices of the highest degrees among the remaining vertices. If $w$ has maximum degree, and $w$ is adjacent to $z$ but not to $x$ among the highest-degree vertices, then there exists $y \in N(x)-N(z)$ since $d(x) \geq d(z)$, and the 2-switch that replaces $w z$ and $x y$ with $w x$ and $z y$ reduces the number of missing desired neighbors of $w$.

Theorem 4 For a positive list $X$ and nonnegative integer $s$, the following are equivalent:

A) $X \in \mathcal{P}_{s+1}$;

B) $X \cup B_{s, n} \in \mathcal{P}_{s}$ for sufficiently large $n$;

C) $t(X) \in \mathcal{P}_{s}$.

Furthermore, $B_{s+1, n} \in \mathcal{P}_{s+1}$, and there are finitely many lists in $\mathcal{P}_{s+1}$ of a given length.

Proof. We prove all claims simultaneously by induction on $s$. Theorem 3 states the equivalence of $\mathrm{A}$ and $\mathrm{C}$ for $s=0$. The definition of $\mathcal{P}_{1}$ yields $\mathrm{A} \Rightarrow \mathrm{B}$ for $s=0$. Note that $B_{1, n} \in \mathcal{P}_{1}$, because $t\left(B_{1, n}\right)=B_{0, n+1} \in \mathcal{P}_{0}$. For every $X \in \mathcal{P}_{1}$ of length $k$, the list $t(X)$ has length at most $2 k$. The finiteness of $\left\{X \in \mathcal{P}_{1}: \ell(X)=k\right\}$ thus follows from the finiteness of the set of graphic lists of length at most $2 k$.

To complete the basis step, it remains only to show $\mathrm{B} \Rightarrow \mathrm{C}$ when $s=0$. Choose some $n$ with $n>\ell(X)$ such that $X \cup B_{0, n}$ is graphic. Note that $n-1$ is the largest value in this 
list. Choose a graph $G$ with degree list $X \cup B_{0, n}$ such that the vertex $w$ of degree $n-1$ is adjacent to vertices of the next highest degrees, as in the proof of the Havel-Hakimi Theorem. Now $G-w$ has degree list $t(X)$.

For the induction step, consider $s>0$.

$\mathrm{A} \Rightarrow \mathrm{B}$. This follows from the definition of $\mathcal{P}_{s+1}$, since part of the final statement of the induction hypothesis is that $B_{s, n} \in \mathcal{P}_{s}$ for all $n$.

$\mathrm{B} \Rightarrow \mathrm{C}$. For sufficiently large $n$, we are given $X \cup B_{s, n} \in \mathcal{P}_{s}$. By the induction hypothesis, $t\left(X \cup B_{s, n}\right) \in \mathcal{P}_{s-1}$. Since $t(X \cup Y)=t(X) \cup t(Y)$ for all $X$ and $Y$, we have $t(X) \cup t\left(B_{s, n}\right) \in \mathcal{P}_{s-1}$. Thus $t(X) \cup B_{s-1, n+1} \in \mathcal{P}_{s-1}$. Since this holds for sufficiently large $n$, the induction hypothesis for $\mathrm{B} \Rightarrow \mathrm{A}$ yields $t(X) \in \mathcal{P}_{s}$.

$\mathrm{C} \Rightarrow \mathrm{A}$. Suppose that $t(X) \in \mathcal{P}_{s}$. By the definition of $\mathcal{P}_{s}$, there exists $n_{0}$ such that $t(X) \cup W \in \mathcal{P}_{s-1}$ whenever $W \in \mathcal{P}_{s-1}$ and $\ell(W)>n_{0}$. Consider $Z=X \cup Y$ for $Y \in \mathcal{P}_{s}$ with $\ell(Y)>n_{0}$. From the final statement of the induction hypothesis, $\ell(Y)>n_{0}$ excludes only finitely many candidates for $Y$ from $\mathcal{P}_{s}$; thus $X \in \mathcal{P}_{s+1}$ will follow from $Z \in \mathcal{P}_{s}$.

We have $t(Z)=t(X) \cup t(Y)$. The induction hypothesis for $\mathrm{A} \Rightarrow \mathrm{C}$ yields $t(Y) \in \mathcal{P}_{s-1}$. Also, $\ell(t(Y)) \geq \ell(Y)>n_{0}$. By the choice of $n_{0}, t(X) \cup t(Y) \in \mathcal{P}_{s-1}$, and hence $t(Z) \in$ $\mathcal{P}_{s-1}$. Now the induction hypothesis for $\mathrm{C} \Rightarrow \mathrm{A}$ implies $Z \in \mathcal{P}_{s}$.

Finally, consider the last statement. We have $t\left(B_{s+1, n}\right)=B_{s, n+1}$, which by the induction hypothesis for this statement belongs to $\mathcal{P}_{s}$. Since we have now proved $\mathrm{C} \Rightarrow \mathrm{A}$, we conclude that $B_{s+1, n} \in \mathcal{P}_{s+1}$. Also, $\mathrm{A} \Rightarrow \mathrm{C}$ and the induction hypothesis for the last statement implies that $\mathcal{P}_{s+1}$ has finitely many lists of a given length.

Recall that the non-graphicality $\gamma(X)$ of an even list $X$ is the least $s$ with $X \in \mathcal{P}_{s}$.

Corollary 5 If $X$ is an even list, then $\gamma(X) \leq \max \left\{0, \frac{\max (X)-\ell(X)+1}{2}\right\}$, with equality for non-graphic lists only when $X$ has only one element larger than 1 . In particular, for $k \geq n-1$ the non-graphicality among $n$-term lists with sum $2 k$ is maximized only by the unique list having just one entry larger than 1 , where it equals $k-n+1$.

Proof. When $X$ is graphic, $\max (X) \leq \ell(X)-1$, so the claim holds when $\gamma(X)=0$. We proceed by induction on $\gamma(X)$.

If $X$ is not graphic, then $\max (X)>1$, and by Theorem 4 we have $\gamma(X)=1+\gamma(t(X))$. Also $\max (t(X))=\max (X)-1$ and $\ell(t(X)) \geq \ell(X)+1$, with equality only when $X$ has exactly one element larger than 1 . By the induction hypothesis, $\gamma(X) \leq \max \{1,1+$ $\left.\frac{\max (X)-1-(\ell(X)+1)}{2}\right\}$, with equality only when $X$ and $t(X)$ each have exactly one element larger than 1. Since $X$ is not graphic, this implies that $\max (X)>\ell(X)-1$, and hence the desired bound and condition for equality follow.

When $X$ has exactly one element larger than 1, the same is true of $t(X)$ (unless the largest element in $X$ is 2), and by induction the bound on $\gamma(X)$ holds with equality. In this case $\max (X)=2 k-n+1$, so the bound $\frac{\max (X)-\ell(X)+1}{2}$ equals $k-n+1$. 


\section{References}

[1] M. Aigner and E. Triesch, Realizability and uniqueness in graphs, Discrete Math. 136 (1994), 3-20.

[2] P. Erdős and T. Gallai, Graphs with prescribed degrees of vertices, Mat. Lapok 11 (1960), 264-274.

[3] S. L. Hakimi, On realizability of a set of integers as degrees of the vertices of a linear graph. J. Soc. Indust. Appl. Math. 10 (1962), 496-506.

[4] V. Havel, Eine Bemerkung ber die Existenz der endlichen Graphen. Časopis Pěst. Mat. 80 (1955), 477-480.

[5] O. Ore, Theory of graphs, Amer. Math. Soc. Colloq. Publ., Vol. XXXVIII, (Amer. Math. Soc., 1962).

[6] G. Sierksma and H. Hoogeveen, Seven criteria for integer sequences being graphic. J. Graph Theory 15 (1991), 223-231. 